Meder, M., Plumbaum, T., \& Hopfgartner, F.

\title{
Perceived and Actual Role of Gamification Principles
}

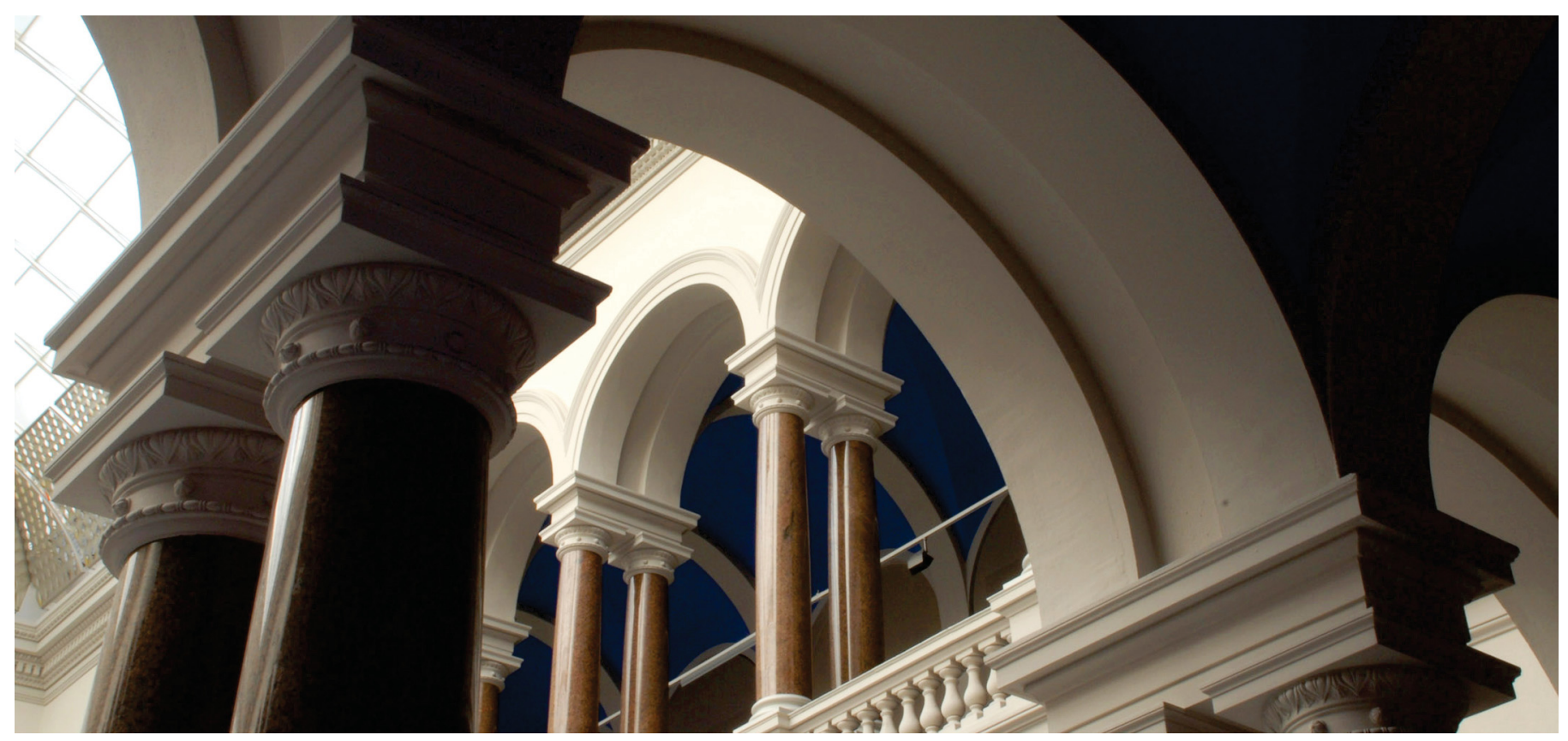

Meder, M., Plumbaum, T., \& Hopfgartner, F. (2013). Perceived and Actual Role of Gamification Principles. In 2013 IEEE/ACM 6th International Conference on Utility and Cloud Computing. IEEE. https://doi.org/10.1109/ucc.2013.95 


\title{
Perceived and Actual Role of Gamification Principles
}

\author{
Michael Meder \\ Till Plumbaum \\ Frank Hopfgartner \\ DAI-Lab, Technische Universität Berlin DAI-Lab, Technische Universität Berlin \\ DAI-Lab, Technische Universität Berlin \\ Email: meder@dai-lab.de \\ Email: till@dai-lab.de \\ Email: hopfgartner@dai-lab.de
}

\begin{abstract}
Although gamification has successfully been applied in office scenarios, it remains unclear how employees really feel about the introduction of a gamified system at their workplace. In this paper, we address this issue from two directions. First, we present the outcome of an online survey where we analyze users' opinion about gamification in a workplace environment. Then, we analyze the interaction logs of a re-designed gamified enterprise bookmarking system to compare the employees' subjective perception of gamification with their actual behavior when using a gamified system. Results indicate that there is a strong relationship between employees' perception of gamification and their actual interaction with such system.
\end{abstract}

\section{INTRODUCTION}

According to Nielsen [12] and Stewart et al. [15], many companies suffer from participation inequality, providing evidence for the assumption that in almost every company, at least one software system exists that can benefit from higher user activity. A promising approach to increase user participation can be achieved by applying gamification methods. Deterding et al. [4] define gamification as "the use of game design elements in non-game contexts", i.e., game mechanics and concepts are applied on non-gaming environments to reach specific goals. Examples for goals include an improvement of user engagement, increased participation, enhanced motivation or just having more fun. Focusing more on the users' perspective, Huotari and Hamari [10] define gamification as " $a$ process of enhancing a service with affordances for gameful experiences in order to support user's overall value creation".

Gamification methods have been applied in various environments and for different purposes such as enterprise workplaces, education, pervasive health care, e-commerce, human resource management and many more (e.g., [1], [3], [13]). Although these studies indicate that gamification can lead to increased user activity, a detailed analysis of users' perception of gamification principles has hardly been studied. We are all individuals and are driven by different input factors such as our personality, as well as social or cultural differences [9], [11], [18], [19], [20]. Especially in an enterprise scenario, it is of uttermost importance to measure challenges and risks that occur due to these differences before introducing gamification methods though. On the one hand, we expect gamification to increase user participation within an enterprise. On the other hand, the visibility of user interaction (or lack thereof), e.g., the position of the employee on a leaderboard can increase the stress level of employees or even cause fear that their activities on a gamified system will be used as an indicator of their engagement with the company.

In this paper, we examine the role of gamification in a workplace environment from an employee's point of view.
More specifically, we aim to answer the following research questions:

- Do employees perceive gamification as positive or negative factor?

- How is the perceived role of gamification reflected by actual usage patterns?

Aiming to address these questions, we defined an online questionnaire on users' expertise and perception of gamification methods, distributed it among employees of a technical research institute and evaluated their responses. In order to evaluate whether their subjective answers are on a par with their actual interaction with a gamified system, we further introduced a gamified Social Enterprise Bookmarking System and analyzed the participants' engagement with this system over a period of one week.

\section{RELATED WORK}

In [2], Dugan et al. describe the transformation of an enterprise bookmarking system into a guessing game called Dogear. In this game, bookmarks and their tags are displayed on screen and the players have to guess, who created this bookmark. If they guess the correct creator of the bookmark, the players can gain points. The Dogear game is inspired by von Ahn's ESP game [17] where users gain points when they use the same tags to describe the content of an image as their teammates. Differing from the ESP game, which exploits "human computation" for the annotation of images, Dogear is focusing on providing methods to learn more about colleagues and their expertise, hence increasing familiarity within a company. They report that within the first month of the release of the system, they had 87 active players from 10 different countries. A detailed analysis is still missing though.

Farzan et al. [7] examine the impact of game mechanics, more precisely the introduction of a points system, on a social enterprise network system (Beehive, IBM). They evaluate the impact of this points system by performing A/B testing, i.e., one half of all users are made aware of the points system, while the other half (i.e., the control group) cannot see this feature. They observe that overall, the introduction of the points system increased the activity level of the users within the system. However, they also report that $72 \%$ of the users in the experimental group never visited the page which describes how to earn points. Besides, they argue that a large portion did not even notice the existence of points.

Addressing this issue further, Farzan et al. [5] also studied if there is any noticeable effect on the usage when the points system is explicitly explained to the users. Therefore, they 
provided further details via email and repeated the experiment. They conclude that points systems can successfully be employed to motivate users to contribute more in an enterprise social network system, especially if combined with email notifications. Further, they conclude that the type of contribution can directly be controlled by the type of gamification applied, i.e., increasing the points for certain types of contributions will indeed result in an increase of contributions of this type. In a follow-up experiment, Farzan et al. [6] increase the social interaction and diversity of content even further by introducing a badge based approach on promoting content. Although they observe an increased activity due to the introduction of gamification methods, the authors argue that they cannot make any statement about the quality of the contributions. Further studies are needed to examine this in detail.

Evaluating the effect of gamification methods from a different perspective, Thom et al. [16] study whether the removal of gamification features from an enterprise social media system has any measurable effect on user activity. They report a significant decline of user activities after removing gamification features, concluding that extrinsic rewards influence user behavior. Interestingly, the authors also noticed some relation between user activity and their geographical location. This supports our premise that there are many factors that can have an impact on the success of workplace gamification.

Hamari [8] evaluates the use of badges in a peer-topeer trading service. They observe that the introduction of gamification mechanisms does not automatically result in an increased use of the system by all users, but that those users, who actively inspect their own badges become more active. This supports our assumption that individual behavior plays an important role in the successful application of gamification methods in an office scenario.

Summarizing, previous research reports an increase of users' activity in an enterprise due to diverse game mechanics. However, these studies also indicate that individual behavior has a significant influence on the success of gamification. To the best of our knowledge, there currently exists no study about employees' perception of gamification. Therefore, we attempt to better understand employees' behavior in more detail.

\section{QUESTIONNAIRE}

In order to address the first research question on employees' perception of gamification principles, we created an online questionnaire where participants were asked to judge various statements on a Five-Point Likert scale. In the remainder of this section, we provide further details about the participating subjects and their responses.

\section{A. Subject recruitment and details}

We recruited participants by sending a brief introduction and a link to the online questionnaire to a mailing list of our research institute at an electrical engineering and computer science department of a major European technical university. Subscribers of this mailing list are over 140 members of this institute, including faculty members, administrative staff, postdoctoral researchers, $\mathrm{PhD}$ students and student research assistants. Given that all subjects are members of a technical research institute, we assume that all participants are highly familiar with using computer systems. To the best of our knowledge, none of the participants has professional experience with gamification in an enterprise setting. In order to participate, subjects had to authenticate using their institute account. We received a response from 53 subjects ( 6 female, 47 male), i.e., over one third of all subscribers of the mailing list. As part of the questionnaire, they were asked to provide their age in a pre-defined range. 23 subjects claim to be between 18-29 years old, 26 subjects are between 30-39 years old, 3 subjects are between 40-49 years old and one to be 50 years or older. Since this distribution roughly matches our institute's age and sex distribution, we argue that the participants are a representative subset of the institute's workforce.

\section{B. Participants' responses}

In the remainder of this section, we introduce all statements that the participants had to answer on a Five-Point Likert scale.

In the first question ( $Q_{1}$ : “contribution"), we asked them to state how often they share or contribute content on enterprise systems such as Wikis or Enterprise CMS. Here, we could observe a rather conservative pattern, i.e., 33.9\% (18 in total) of all subjects said that they sometimes contribute content, $33.9 \%$ (18) said that they seldom contribute and $11.3 \%$ (6) never share or contribute on such systems. Only $20.7 \%$ said that they often (8) or very often (3) contribute content. These answers are in line with our own (subjective) observations and the analysis of Wikipedia contributions by Ortega et al. [14] showing that content is often contributed by few individuals.

After asking the subjects to assess their current contribution and share activities on enterprise systems, the subjects were asked in question ( $Q_{2}$ : "familiarity"), to state how familiar they are with the term "gamification". This was also the first time we mentioned the term gamification in the questionnaire. $66 \%$ of all participants claimed that they were either to a great extent (10 subjects) or somewhat (25) familiar with it. 14 subjects said that they have very little knowledge about it while four subjects were not familiar with it at all. We conclude from these responses that there is a general awareness of gamification principles (especially amongst those colleagues who closely work with the authors of this paper), while many lack further details to describe these principles.

In the following question $Q_{3}$ ("motivation"), we were further interested in their own attitude towards gamification. Therefore, we asked them to judge whether gamification would motivate them to participate even more in enterprise systems. In order to guarantee that all subjects had the same understanding of gamification, we also provided a brief definition (as stated on Wikipedia) that should help to better understand this question. $43 \%$ of all participants stated that they were undecided on how to judge this statement. $34 \%$ of them agreed to this statement while $22 \%$ disagreed (9) or strongly disagreed (3). Although only a few more than those who disagree tends to agree with this statement. With $43 \%$ answered undecided it is evident that the subjects are not very convinced of the role of gamification in an enterprise environment. This goes in line with $Q_{2}$ which indicates that the subjects are not too familiar with the concept.

In the next question ( $Q_{4}$ : "positive effects"), we asked the participants to state whether game mechanics like points, 
badges and leader boards have a positive effect on the enterprise and its staff. While $33.9 \%$ were undecided, a majority of over $50 \%$ either agree (26) or strongly agree (1) with this statement. Only eight participants disagreed (4) or strongly disagreed (4). This distribution of judgements seems to indicate that the majority of participants have a rather positive perception of gamification principles.

In the last question ( $Q_{5}$ : "negative effects"), we wanted to know whether the participants believe that there are negative effects on the enterprise and its staff caused by game mechanics like points, badges and leaderboards. Here, a clear preference can not be observed. $49 \%$ of all subjects were undecided, $22.6 \%$ either agreed or disagreed with this statement while only 2 participants strongly agreed with it.

Summarizing, in this section, we aimed to evaluate whether gamification is seen positively or negatively within an enterprise context. The analysis of our online questionnaire revealed that although many participants of the study are (to some extent) familiar with gamification and are convinced that it can have a positive effect on an enterprise and its staff, they are not convinced that it can serve as intrinsic motivation for themselves to contribute more on enterprise systems. Addressing this question further, we present a gamified enterprise tagging system in the next section which shall shed further light on the difference between the perceived and the actual role of gamification on users' behavior in an enterprise setting.

\section{A GAMIFIED ENTERPRISE TAGging System}

Following our first research question on employees' perception of gamification, we were further interested in comparing this perceived role with users' actual interaction with a gamified system. In the remainder of this section, we first introduce such a system, i.e., an enterprise bookmarking system. Then, we introduce the gamification elements that have been added to the system. An evaluation of the users' interaction with this system is provided in the next section.

\section{A. Legacy Enterprise Bookmarking System}

In 2009, we developed a social bookmarking system (Figure 1) in close cooperation with a large company. Social bookmarking systems became popular in the early years of this century with the rise and success of Delicious ${ }^{1}$. The main idea of such systems is that bookmarks can be annotated using tags and shared with others. Providing similar functionalities as the well-known Delicious system, our system additionally allows to create bookmarks for files on internal file server, taking into account existing rights management and the possibility to share bookmarks not only with other people but also with people having a certain tag.

The system has two main views, the personal site showing all bookmarks of a user, public and private ones (a user can mark bookmarks as private being only visible to them and not appearing in public searches) and a general view, called 'What's new' showing all public bookmarks. Bookmarks and users can both be tagged and searched. The system also offers a set of tools to ease the bookmarking process. We developed a JavaScript based bookmarklet, allowing people to easily

\footnotetext{
${ }^{1}$ https://delicious.com
}

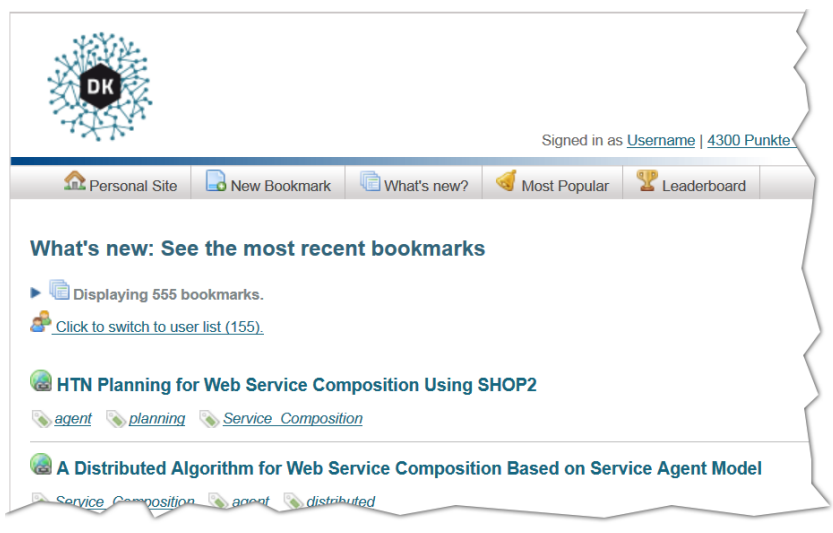

Fig. 1: The main view of the bookmarking system, displaying all publicly bookmarked items.

bookmark web pages and a Windows tool to bookmark files. Also integrated is an automatic tags recommendation system recommending tags based on the bookmarked item (extracting the most important words from the describing text) and the most used tags by the user. We also integrated a so-called "Most Popular" section, showing the most frequent used tags of the week and of all time to help users see what topic is currently trending in the company.

\section{B. Gamification Elements}

In the context of this study, the existing system was carefully extended with three well known and researched gamification elements - Points, Badges, and a Leaderboard. The three elements were chosen to cover different types of motivation and Gamfication types. Rewards, with points and badges, for fulfilling different tasks, and a competition element with the leaderboard. While the points reward direct interaction with the system such as creating a new bookmark, badges are given for completing longer term goals such as continuously creating new bookmarks in a certain period of time. How to achieve points and badges is explained to the user by detailed descriptions of which actions score what points and what is needed to achieve a badge. The leaderboard, accessible only after the user logged in, is integrated in the main menu and within easy reach for the user. In the remainder of this section, we introduce the applied gamification elements in more detail.

Points: Almost every user interaction with the bookmarking system gives points, including daily login (200 points), adding a public/private bookmark (100/25 points), adding a bookmark with at least one tag ( 25 points), accepting a bookmark recommendation (200 points) and many others.

Badges: In the gamified bookmarking system, we introduced five different types of badges. For getting points, creating bookmarks, loyalty (regular usage), and positive recommendations.

Leaderboard: In the leaderboard, users are ranked by points in decreasing order. Besides, achieved badges are displayed to promote them further. The board shows two rankings: the monthly leaderboard on the left-hand side which will be automatically reset every month and the all time leaderboard on the right-hand side. This is done to avoid frustrating new users and to create a new challenge every month. 
Feedback: To ensure that the user is aware of points and badges, we integrated a message system (Figure 2). From the first log in, users will get a small onsite popup message, also called toast message. These messages appear every time a user got a reward by points or badges and if the user reached a higher rank on the leaderboard. Moreover, the user gets a message when another user copied one of her bookmarks or accepted a bookmark recommendation. After ten seconds, the messages disappear automatically.

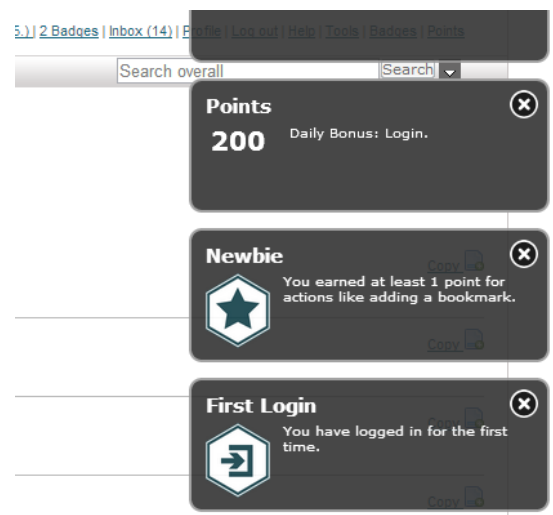

Fig. 2: Messages appearing on the user's first log in.

\section{EXPERIMENT AND RESULTS}

In order to evaluate our second research question whether users' perception of gamification (as stated in the online questionnaire) matches with their behavior using a gamified system, we announced the relaunch of the gamified enterprise bookmarking system using the institute-wide mailing list and observed the users' interaction with the system. In this section, we provide an overview of the systems usage from 2009 till the introduction of gamification functionalities in mid June 2013 to provide insights into the usage of the system before it got gamified. Following that, we present the usage statistics after the gamified version was introduced.

\section{A. System Usage Before Gamification}

In the analysis of usage patterns we look at data from 2009 until the gamified version went online in June $2013^{2}$. The focus of the analysis is to look at two important aspects of the bookmarking system: The main feature, the creation of bookmarks, and the number of recommended bookmarks to colleagues, one of the main goals for enterprises to support knowledge sharing. Since a user-centric evaluation of this system was never intended for the legacy system, the (pregamified) system did not track any user interactions. Thus, only actions resulting in a database entry can be tracked. This was changed in the gamified version.

Table I shows the yearly distribution of created items and active users. Outliers, in this case a power user responsible for half of all system interactions, are removed from the statistics. After a solid start in 2009 with roughly one third of employees using the system, 2010 already showed a significant decline in

\footnotetext{
${ }^{2}$ The gamified version went online on June, 19th. The presented analysis however only considers data till June, 1st, to prevent any influence of the data because of any office kitchen chatter about the bookmarking system.
}

the usage. While the usage number remained stable for the years 2010-2013, only 3\% of people were using the system.

\begin{tabular}{cccccc}
\hline & 2009 & 2010 & 2011 & 2012 & $2013^{*}$ \\
\hline Bookmarks & 291 & 29 & 30 & 13 & 26 \\
Users & 30 & 4 & 4 & 3 & 5
\end{tabular}

TABLE I: Created items per year and the number of active users $(*=$ until June $1 \mathrm{st})$.

Table II shows the total number of bookmark recommendations. Similar to the created items, the number peaked in 2009. From 2009 till 2013, they remained on a stable level. This comes as no surprise, as the system's user interface (UI) supports the recommendation during a bookmark creation. Recommending an item at a later stage was still possible, but not as compelling as in the creation process. Noticeable is the comparison of the number of users. As shown in Table I, we recorded a total of 30 users in 2009 with only 14 of them using the recommendation feature. This might be due to the mentioned UI flaws, but could also indicate that those users use the system only for personal managing purposes. From 2010 till 2013 usage numbers remain the same, suggesting that those users who use the system over a long period of time are well familiar with its functionalities.

\begin{tabular}{cccccc}
\hline & 2009 & 2010 & 2011 & 2012 & $2013^{*}$ \\
\hline Recommendations & 61 & 34 & 31 & 26 & 18 \\
Users & 14 & 4 & 4 & 3 & 5 \\
\hline
\end{tabular}

TABLE II: Recommended bookmarks per year and the number of active users $(*=$ until June 1 st).

The statistics show that the non-gamified version of the bookmarking system was used only rarely. In the next section, we present and discuss results of the gamified version of the bookmarking system. Then, we compare users' feedback from the questionnaires with the actual usage patterns to answer our second research question. Finally, we discuss limitations and shortcomings of the presented study and discuss future steps for gamification in enterprises.

\section{B. System Usage After Gamification}

As mentioned above, we tracked user interaction to analyze their behavior while interacting with the gamified system. For tracking their behavior, we relied on the Open Web Analytics ${ }^{3}$ platform which captures users' interaction with all HTML elements of the system. Using this software, we respect the "do not track" option that can be set in the browser, resulting in incomplete or no web tracking data for some users of the system. Besides, we had to interrupt the experiment shortly after announcing the re-designed system due to a software bug. Two days later, we re-announced the system. All user interactions that took place within these two days has been omitted from this evaluation.

After announcing the system, a total of 18 users registered with the system. Seven of them, however, did not use the system at all. 14 of the registered users also participated in the online questionnaire, nine of these users logged in

\footnotetext{
${ }^{3}$ http://www.openwebanalytics.com/
} 


\begin{tabular}{|c|c|c|c|c|c|}
\hline \multicolumn{3}{|c|}{ leaderboard } & \multicolumn{3}{|c|}{ \# page requests } \\
\hline \multirow[b]{2}{*}{ rank } & \multirow[b]{2}{*}{ points } & \multirow[b]{2}{*}{ \# badges } & \multirow[b]{2}{*}{ all } & \multicolumn{2}{|c|}{ has game design elements } \\
\hline & & & & leaderboard & other \\
\hline 1 & 5700 & 12 & 130 & 58 & 7 \\
\hline 2 & 4300 & 9 & 230 & 22 & 14 \\
\hline 3 & 2625 & 9 & - & - & - \\
\hline $4 *$ & 2100 & 9 & - & - & - \\
\hline 5 & 1675 & 7 & 51 & 0 & 13 \\
\hline 6 & 1360 & 8 & 47 & 5 & 7 \\
\hline 7 & 1150 & 5 & 24 & 2 & 0 \\
\hline 8 & 1125 & 7 & - & - & - \\
\hline 9 & 450 & 3 & 15 & 0 & 0 \\
\hline 10 & 450 & 4 & 7 & 1 & 2 \\
\hline $11^{*}$ & 450 & 3 & 17 & 3 & 6 \\
\hline $12 . .19$ & 200 & 2 & & & \\
\hline
\end{tabular}

TABLE III: Leaderboard one week after email announcement of the gamified bookmarking system $(*=$ user did not fill in the questionnaire, - = incomplete or no tracking data).

more than once. One user received a badge and 200 points for successfully recommending a document to another user. This user has left the institute over two years ago, i.e., he recommended this document a long time ago. Obviously, this user did not participate in the online questionnaire.

Table III provides an overview of the users' activities with the system. They are ranked based on their position in the leaderboard after one week. Those users who are annotated with the * symbol did not fill in the online questionnaire. It can be seen, for example, that after eight days (from Wed to Wed) of use, the user with rank $1\left(U_{\text {rank }_{1}}\right)$ gained a total of 5700 points, collected 12 badges, visited the leaderboard 58 times and other pages with gamification elements 7 times. In total, the user visited pages containing game design elements more than twice as often as the other users. Besides, $U_{\text {rank }_{1}}$ participated in the online questionnaire.

Based on these interactions, we categorize the users into four different types: The Top 3 users, a midfield (positions 4-8 in the leaderboard), users who use the system for a very short time only (positions 9-11 in the leaderboard) and Users 12-19 who logged into the system only once.

One question is how these users interacted with the system in detail. Table IV shows the interaction of all users at different days of the experiment. As expected, the most interactions were recorded in the first few days of the experiment. Every day, the leaderboard was the most visited gamified page of the system. During the weekend, i.e., Day $4 \& 5$ of the experiment, no direct interaction with the gamification elements was recorded. The overall number of page requests declines over the course of the experiment, suggesting that the overall interest in the system declined as well.

\section{COMPARISON}

After providing an overview of the system usage before and after the system got gamified, we discuss in this section the user interaction with respect to their feedback in the online

\begin{tabular}{lcc}
\hline & $\begin{array}{c}\text { Group A } \\
(\# \operatorname{login}>0)\end{array}$ & $\begin{array}{c}\text { Group B } \\
(\text { \# } \operatorname{login}=0)\end{array}$ \\
\hline$Q_{1}$ (contribution) & $\mathbf{3 . 0 0}$ & 2.50 \\
$Q_{2}$ (familiarity) & $\mathbf{3 . 0 7}$ & 2.56 \\
$Q_{3}$ (motivation) & $\mathbf{3 . 2 9}$ & 2.94 \\
$Q_{4}$ (positive) & $\mathbf{3 . 6 4}$ & 3.11 \\
$Q_{5}$ (negative) & 2.86 & $\mathbf{3 . 2 0}$ \\
\hline
\end{tabular}

TABLE V: Mean average answers in online questionnaire of users who participated only in the questionnaire (Group B) and users who, in addition, logged in at least once in the gamified bookmarking system (Group A). (higher value indicates higher assessment, higher frequency and stronger agreement)

questionnaire, hence addressing the research question whether the perceived role of gamification is reflected by actual usage.

Table V shows the mean average answers of all users who participated in the online questionnaire. In the first rows of the table, we segment this group in two parts: those users who logged into our system at least once (Group A, where \# $\operatorname{login}>0$ ) and those who did not $\log$ in (Group B, where \# login = 0). As expected, members of Group A reported a higher familiarity (3.07 vs. 2.56) with gamification than Group B. Further, they also (on average) stated a higher content contribution (3.00 vs. 2.50$)$ to online systems, stated that gamification can result in higher motivation (3.29 vs. 2.94) and believed more in the positive effect of gamification (3.64 vs. 3.11) than their colleagues from Group B. Beyond that, the members of Group B had (on average) a stronger opinion about the negative effects than their colleagues from Group A. This seems to indicate that those employees who have a rather positive impression of gamification are also more likely to use such system at least once.

In order to further study whether this positive attitude is also reflected in the users' constant use of the system, i.e., addressing our second research question, we split Group A further into two sub groups: the top eight users (according to the leaderboard) and the remaining 9 users who logged in at least once. Their corresponding answers are shown in Table VI. Surprisingly, the Top 8 users are more aware of negative effects caused by gamification than the rest. Similar to Table V, we can observe a higher frequency, higher assessment and stronger agreement by those employees who were more active on the gamified system, i.e., those users to ended up on higher positions in the monthly leaderboard. This indicates that the perceived role of gamification is indeed reflected by actual user interaction with a gamified system, hence answering our second research question.

\section{CONCLUSION}

In this paper, we studied the perceived and actual role of gamification in a workplace environment. We focused on two questions.

First, we were interested to know whether employees perceive gamification as positive or negative factor in an enterprise. Therefore, we distributed an online questionnaire amongst members of a large research institute where participants were asked to judge and respond to different statements and questionnaires on a Five-Point Likert scale. Their 


\begin{tabular}{|c|c|c|c|c|c|c|c|c|c|}
\hline & \multirow[b]{2}{*}{ day } & \multicolumn{2}{|c|}{ \# bookmarks } & \multicolumn{6}{|c|}{ \# request all pages and pages with game design elements } \\
\hline & & create/copy & recommend & all & leaderboard & my points & my badges & help points & help badges \\
\hline 1 & (Wed) & 13 & 1 & 371 & 32 & 19 & 9 & 6 & 11 \\
\hline 2 & (Thu) & 25 & 1 & 135 & 26 & 1 & 0 & 0 & 0 \\
\hline 3 & (Fri) & 11 & 6 & 126 & 12 & 3 & 4 & 1 & 1 \\
\hline $4-5$ & (Sat/Sun) & 0 & 0 & 7 & 0 & 0 & 0 & 0 & 0 \\
\hline 6 & (Mon) & 4 & 3 & 72 & 16 & 0 & 0 & 0 & 1 \\
\hline 7 & (Tue) & 4 & 0 & 58 & 10 & 3 & 1 & 1 & 1 \\
\hline 8 & (Wed) & 9 & 0 & 32 & 4 & 3 & 0 & 0 & 2 \\
\hline
\end{tabular}

TABLE IV: Bookmark contributions and page request over time.

\begin{tabular}{lcc}
\hline & $\begin{array}{c}\text { Group } A_{1} \\
(\text { rank } \leqslant 8)\end{array}$ & $\begin{array}{c}\text { Group } A_{2} \\
(\text { rank }>8)\end{array}$ \\
\hline$Q_{1}$ (contribution) & $\mathbf{3 . 1 4}$ & 2.86 \\
$Q_{2}$ (familiarity) & $\mathbf{3 . 1 4}$ & 3.00 \\
$Q_{3}$ (motivation) & $\mathbf{3 . 7 1}$ & 2.86 \\
$Q_{4}$ (positive) & $\mathbf{3 . 7 1}$ & 3.57 \\
$Q_{5}$ (negative) & $\mathbf{3 . 1 4}$ & 2.57 \\
\hline
\end{tabular}

TABLE VI: Mean average answers in online questionnaire of top eight users (Group $A_{1}$ ) of the leaderboard and users on position 9-19 (Group $A_{2}$ ). (higher value indicates higher assessment, higher frequency and stronger agreement)

responses indicate that although some employees were already familiar with the idea of gamification and are convinced that it can have a positive effect on their work. Nevertheless, a majority of participants stated that they are not convinced that it can serve as intrinsic motivation for themselves.

In our second research question, we were interested to evaluate whether this perceived role of gamification that is reported by the participants of the online questionnaire matches their actual behavior when using a gamified system. Therefore, we gamified an existing enterprise bookmarking system and introduced it in the same research institute. After one week, we analyzed the online questionnaire based on the users' interaction with this system. We observed that those employees who showed a positive tendency towards gamification also interact more with the gamified system. Therefore, we conclude that there is a relationship between the perceived and the actual role of gamification principles in a workplace environment.

The system which was introduced in this paper is still in use. As future work, we intend to analyze employees' interaction over a longer period of time and hopefully a larger number of active users.

\section{ACKNOWLEDGMENT}

The authors would like to thank Eugen Rein and Christian Sauer for programming and design support.

\section{REFERENCES}

[1] Anderson, A., Huttenlocher, D., Kleinberg, J., and Leskovec, J. Steering user behavior with badges. In Proc. $W W W$ (Rio de Janeiro, Brazil, 2013), 95-106.

[2] Casey Dugan, Michael Muller, David R. Millen, Werner Geyer, Beth Brownholtz, M. M. The dogear game: a social bookmark recommender system. In Proc. ACM GROUP'07 (2007), 387-390.
[3] Cheng, L.-T., Shami, S., Dugan, C., Muller, M., DiMicco, J., Patterson, J., Rohal, S., Sempere, A., and Geyer, W. Finding Moments of Play at Work. In Workshop on Gamification: Using Game Design Elements in Non-Gaming Contexts (2011), 2-5.

[4] Deterding, S., Dixon, D., Khaled, R., and Nacke, L. From game design elements to gamefulness: defining gamification. Proc. Int. Academic MindTrek Conference (2011), 9-15.

[5] Farzan, R., and DiMicco, J. When the experiment is over: Deploying an incentive system to all the users. In Persuasive Technology, ACM (2008).

[6] Farzan, R., DiMicco, J., and Brownholtz, B. Spreading the honey: a system for maintaining an online community. Proc. ACM GROUP'09 (2009), 31-40.

[7] Farzan, R., DiMicco, J., and Millen, D. Results from deploying a participation incentive mechanism within the enterprise. In Proc. SIGCHI conference on Human factors in computing systems (2008), 563-572.

[8] Hamari, J. Transforming Homo Economicus into Homo Ludens: A Field Experiment on Gamification in A Utilitarian Peer-to-Peer Trading Service. Electronic Commerce Research and Applications, 12 (2013).

[9] Hamari, J., and Koivisto, J. Social motivations to use gamification: An empirical study of gamifying exercise. Proc. ECIS'13 (2013), 1-12.

[10] Huotari, K., and Hamari, J. Defining gamification: a service marketing perspective. Proceeding of the 16th International Academic MindTrek Conference (2012), 17-22.

[11] Khaled, R. It's Not Just Whether You Win or Lose: Thoughts on Gamification and Culture. In Workshop on Gamification: Using Game Design Elements in Non-Gaming Contexts (2011), 1-4.

[12] Nielsen, J. Participation Inequality: Encouraging More Users to Contribute, 2006.

[13] Nikkila, S., Lin, S., Sundaram, H., and Kelliher, A. Playing in Taskville: Designing a Social Game for the Workplace. In Workshop on Gamification: Using Game Design Elements in Non-Gaming Contexts (2011), 1-4.

[14] Ortega, F., Gonzalez-Barahona, J., and Robles, G. On the inequality of contributions to wikipedia. In Proc. Int. Conference on System Sciences (2008), 304-304.

[15] Stewart, O., Lubensky, D., and Huerta, J. Crowdsourcing participation inequality: a SCOUT model for the enterprise domain. In Proceedings of the ACM SIGKDD Workshop on Human Computation (2010), 30-33.

[16] Thom, J., Millen, D., and DiMicco, J. Removing gamification from an enterprise SNS. Proc. CSCW'12 (2012), 1067-1070.

[17] von Ahn, L., and Dabbish, L. Labeling images with a computer game. Proc. CHI '04 6, 1 (2004), 319-326.

[18] Yang, J., Morris, M. R., Teevan, J., Adamic, L. A., Ackerman, M. S., and Way, O. M. Culture Matters : A Survey Study of Social Q \& A Behavior. In International AAAI Conference on Weblogs and Social Media (2011), 409-416.

[19] Yee, N. Motivations for play in online games. CyberPsychology \& Behavior (2006), 772-775.

[20] Yee, N., Ducheneaut, N., and Nelson, L. Online gaming motivations scale: development and validation. Proc. CHI '12 (2012), 2803-2806. 